\title{
An integrative genomic analysis identifies Bhmt2 as a diet-dependent genetic factor protecting against acetaminophen-induced liver toxicity
}

\author{
Hong-Hsing Liu, ${ }^{1,7,8}$ Peng Lu, ${ }^{1,7}$ Yingying Guo, ${ }^{1}$ Erin Farrell, ${ }^{1}$ Xun Zhang, ${ }^{1}$ \\ Ming Zheng, ${ }^{2}$ Betty Bosano, ${ }^{2}$ Zhaomei Zhang, ${ }^{1}$ John Allard, ${ }^{1}$ Guochun Liao, ${ }^{1}$ Siyu Fu, ${ }^{1}$ \\ Jinzhi Chen, ${ }^{1}$ Kimberly Dolim, ${ }^{2}$ Ayako Kuroda, ${ }^{2}$ Jonathan Usuka, ${ }^{2}$ Janet Cheng, ${ }^{1}$ \\ William Tao, ${ }^{2}$ Kevin Welch, ${ }^{3}$ Yanzhou Liu, ${ }^{4}$ Joseph Pease, ${ }^{4}$ Steve A. de Keczer, ${ }^{4}$ \\ Mohammad Masjedizadeh, ${ }^{4}$ Jing-Shan Hu, ${ }^{1}$ Paul Weller, ${ }^{2}$ Tim Garrow, ${ }^{5}$ and Gary Peltz ${ }^{6}$ \\ ${ }^{1}$ Department of Genetics and Genomics, Roche Palo Alto, Palo Alto, California 94304, USA; ${ }^{2}$ Non-Clinical Safety, Roche Palo Alto, Palo \\ Alto, California 94304, USA; ${ }^{3}$ Technical Sciences, Roche Palo Alto, Palo Alto, California 94304, USA; ${ }^{4}$ Laboratory of Molecular \\ Immunology, National Heart, Lung, and Blood Institute, National Institutes of Health Bethesda, Maryland 20892, USA; ${ }^{5}$ Department \\ of Food Science and Nutrition, University of Illinois, Urbana, Illinois 61801, USA; ${ }^{6}$ Department of Anesthesia, Stanford University, \\ Stanford, California 94305, USA
}

\begin{abstract}
Acetaminophen-induced liver toxicity is the most frequent precipitating cause of acute liver failure and liver transplant, but contemporary medical practice has mainly focused on patient management after a liver injury has been induced. An integrative genetic, transcriptional, and two-dimensional NMR-based metabolomic analysis performed using multiple inbred mouse strains, along with knowledge-based filtering of these data, identified betaine-homocysteine methyltransferase 2 (Bhmt2) as a diet-dependent genetic factor that affected susceptibility to acetaminophen-induced liver toxicity in mice. Through an effect on methionine and glutathione biosynthesis, Bhmt2 could utilize its substrate (S-methylmethionine $[\mathrm{SMM}]$ ) to confer protection against acetaminophen-induced injury in vivo. Since SMM is only synthesized in plants, Bhmt2 exerts its beneficial effect in a diet-dependent manner. Identification of Bhmt2 and the affected biosynthetic pathway demonstrates how a novel method of integrative genomic analysis in mice can provide a unique and clinically applicable approach to a major public health problem.
\end{abstract}

[Supplemental material is available online at http://www.genome.org. The microarray data from this study have been submitted to the NCBI Gene Expression Omnibus (http://www.ncbi.nlm.nih.gov/geo) under accession no. GSE17649.]

\begin{abstract}
Acetaminophen (APAP) is the most widely used analgesic in the United States; it is a safe and effective drug when administered appropriately. However, an acute overdose causes liver damage by inducing localized centrilobular cell death (Bessems and Vermeulen 2001; James et al. 2003). Because of its widespread use and low therapeutic index, APAP toxicity has become the most frequent cause of acute liver failure (Perkins 2006). APAP is primarily metabolized by sulfation and glucuronidation; the resulting conjugates (APAP-Glu or APAP-Sul) are excreted in urine and bile (Chen et al. 2003). However, cytochrome P450 (CYP450) enzymes oxidize a small portion of APAP to a reactive quinone metabolite (N-acetylp-benzoquinone imine, NAPQI), which covalently binds to cysteine residues in more than 50 different cellular proteins. These adducts are believed to interfere with critical cellular functions, leading to cell death and cell lysis (James et al. 2003). These in turn would induce immunological responses that are important in shaping the final outcome (Masson et al. 2008; Harrill et al. 2009; Masubuchi et al. 2009). At therapeutic doses, the reactive quinone
\end{abstract}

\footnotetext{
${ }^{7}$ These authors contributed equally to this work.

${ }^{8}$ Corresponding author.

E-mail honghsing.liu@gmail.com; fax (650) 721-2420.

Article published online before print. Article and publication date are at http://www.genome.org/cgi/doi/10.1101/gr.097212.109.
}

metabolite can be efficiently detoxified by conjugation to glutathione (GSH), which plays a major role in detoxifying many hepatotoxic drugs (Meister and Anderson 1983). However, after a toxic dose of APAP, hepatic glutathione can be depleted by as much as $90 \%$, enabling the reactive intermediates to form adducts with cellular proteins, which leads to hepatic cell death (James et al. 2003). N-acetylcysteine has been shown to be the most effective antidote when administered to patients with severe and ongoing APAP poisoning; and it has been shown to act via GSH repletion (Chun et al. 2009). Thus, maintaining hepatic GSH levels at an early stage is critical for limiting APAP-induced liver toxicity.

Many factors have been associated with susceptibility to APAPinduced liver injury, including: factors affecting metabolic activation or GSH-mediated detoxification (Bai and Cederbaum 2004; Guo et al. 2004; Jia et al. 2005; Welch et al. 2005), the immune response and cytokine production (Hogaboam et al. 2000; Ishida et al. 2002, 2004; Liu et al. 2004), or enzymes regulating apoptosis (Limaye et al. 2003, 2006; Bhave et al. 2008). Global analyses of APAP-induced changes in gene expression (Reilly et al. 2001) or cellular proteins (Qiu et al. 1998; Ruepp et al. 2002; Welch et al. 2005) have also identified a vast array of other potential mediators. Unfortunately, none of these factors have been actually enabled strategies that could be proactively used to prevent APAP-induced liver injury. 
To better characterize genetic factors affecting susceptibility to APAP-induced liver toxicity, we performed an integrated genetic, transcriptional, and metabolomic analyses using a panel of inbred mouse strains. Two-dimensional (2D) NMR-based metabolomic analysis was used to characterize changes in endogenous hepatic metabolites in susceptible and resistant mouse strains, and APAP-induced changes in hepatic gene expression were simultaneously measured using microarrays. The transcriptional and metabolomic data were jointly analyzed, and functional information within the Gene Ontology database was used to identify a subset of genes that could affect susceptibility to APAPinduced liver injury. These results indicate that multiple genetic loci and an interacting network of metabolic factors affect susceptibility to APAP-induced liver injury, and that genetic variation altering BHMT2 enzyme activity was one of the genetic susceptibility factors. Its substrate (S-methylmethionine [SMM]) provided strain-dependent protection against APAP-induced liver injury in vivo. Since SMM is synthesized only in plants that are eaten by mammals (Mudd and Datko 1990), Bhmt2 belongs to a new class of host factors with a diet-dependent effect on susceptibility to APAPinduced liver injury.

\section{Results}

\section{Differential susceptibility to APAP-induced hepatotoxicity}

The susceptibility of 16 inbred mouse strains to the liver toxic effect of APAP was assessed by measuring the serum alanine aminotransferase (ALT) activity after a single administration of (300 mg/kg i.p.) APAP. This dose was previously shown to cause liver toxicity in mice (Welch et al. 2005). Significant liver injury developed at $6 \mathrm{~h}$ in all strains, except SJL/J (Fig. 1A). The resistant phenotype of SJL/J mice is consistent with an earlier report (Welch et al. 2005).

We then performed a detailed analysis of the hepatic response to APAP toxicity in the resistant (SJL/J) and in thrill sensitive (C57BL/6J, DBA/2J, and SM/J) strains. The serum ALT level, veterinarian-determined moribund status, and hepatic histopathology were all used as indices of toxicity. Six hours after APAP treatment, all three sensitive strains had significantly elevated serum ALT levels. Within $48 \mathrm{~h},>60 \%$ of the mice from the three sensitive strains were in a moribund state, while all SJL/J mice survived (Fig. 1B). Although the magnitude of the serum ALT elevations varied among different experiments, C57BL/6J was reproducibly the most susceptible strain, as evidenced by the fact that all were moribund by $6 \mathrm{~h}$ after dosing. Veterinarians that were blinded as to whether APAP or vehicle was administered periodically assessed morbidity according to predetermined criteria during this experiment, and moribund mice were euthanized as required by the animal protocol. Liver histology was consistent with the biochemical and morbidity data. SJL/J mice had relatively minor drug-induced liver injury, while there was extensive centrilobular necrosis in livers obtained from the three sensitive strains (Fig. 1C,D; data not shown).

\section{Metabolomic and pharmacokinetic analyses}

Our previously published method for ${ }^{1} \mathrm{H}^{13} \mathrm{C} 2 \mathrm{D}$ NMR (Zheng et al. 2007) analysis was used to compare the metabolomic changes in livers obtained from the three sensitive (C57BL/6J, DBA/2J, SM/J) strains with those in the resistant (SJL/J) strain at 0,3 , and $6 \mathrm{~h}$ after APAP exposure. Principal component analysis (Duda et al. 2001) indicated that the metabolite profiles obtained $3 \mathrm{~h}$ after drug

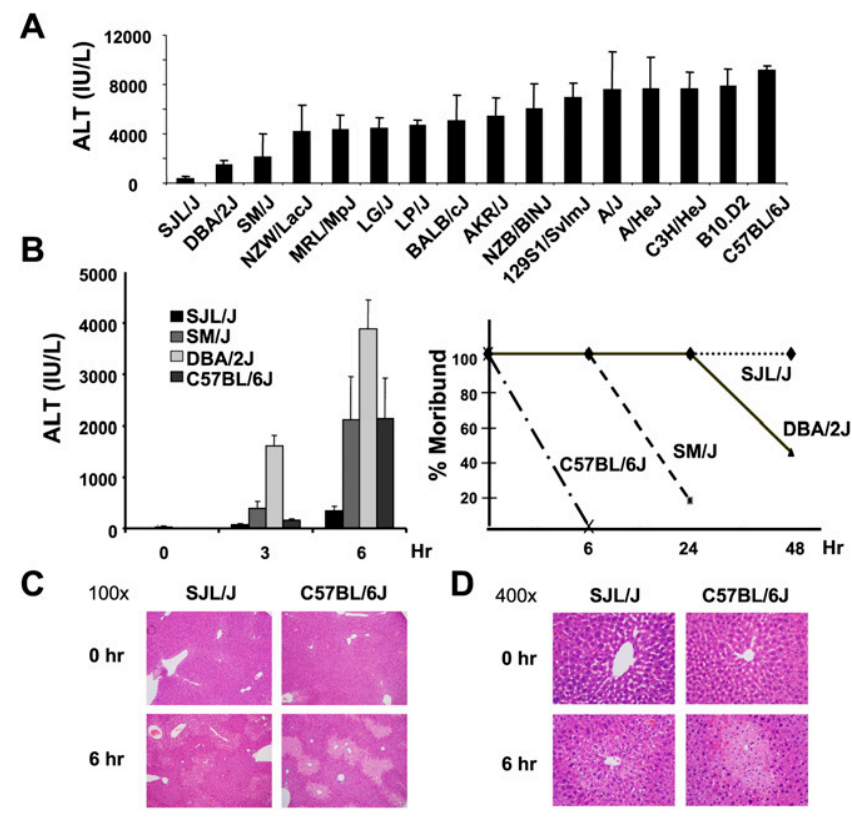

Figure 1. Acetaminophen (APAP)-induced hepatotoxicity in inbred mouse strains. $(A)$ Serum alanine aminotransferase (ALT) activity was measured $6 \mathrm{~h}$ after 16 inbred mouse strains were treated with 300 i.p. mg/kg APAP. The bars represent the average \pm SD for $N=5$ mice per group. SJL/J mice were resistant to APAP-induced toxicity, while all other strains developed significant toxicity. (B) Serum ALT (left) and the percentage of moribund mice (right) were measured at the indicated times after APAP treatment in four inbred strains. Veterinarians that were blinded to whether the mice received drug or placebo determined when mice were moribund. The ALT measurements are the average $\pm S E(N=7$ mice per group); and the percentage of moribund mice was determined from analyses of 10 mice. $(C, D)$ Hematoxylin and eosin staining of liver tissue from SJL/J and C57BL/6J mice at 0 and $6 \mathrm{~h}$ after APAP treatment are shown at $100 \times$ and $400 \times$ magnifications, respectively.

treatment differentiated the resistant SJL/J mice from the three sensitive strains (Supplemental Fig. 1; Supplemental Table 2). Four endogenous metabolites (GSH, alanine, lactate, and betaine) had a unique abundance pattern in the resistant strain, which were qualitatively and quantitatively distinct from those in the three sensitive strains (Fig. 2A). It was of particular interest that SJL/J mice uniquely maintained their hepatic GSH level $3 \mathrm{~h}$ after APAP administration, while C57BL/6J mice, which had the highest morbidity, had the most sustained decrease in hepatic GSH. Therefore, this unbiased metabolomic analysis indicates that hepatic GSH abundance was one factor that distinguished the resistant SJL/J strain from the susceptible strains.

The resistant phenotype of SJL/J mice could be due to an inability to produce reactive NAPQI metabolites. To investigate this possibility, the amount of APAP and five major metabolites in bile, plasma, urine, and liver at 1 and $2 \mathrm{~h}$ after APAP administration were measured in a susceptible (B10.D2) and a resistant (SJL/J) strain (Fig. 2A; Supplemental Table 1). At $1 \mathrm{~h}$, there were no significant differences in any drug metabolites in bile or urine; and the serum levels of the metabolites were comparably low in both strains (Fig. 2B; Supplemental Table 1). These findings suggest that APAP was similarly biotransformed and excreted in SJL/J and B10.D2 mice in the early phase. Alterations in the biliary concentrations of APAP metabolites have been used to identify differences in APAP biotransformation (Dai et al. 2006). The biliary concentrations of APAP-GSH and APAP-Cysteine (APAP-CYS) in 
A

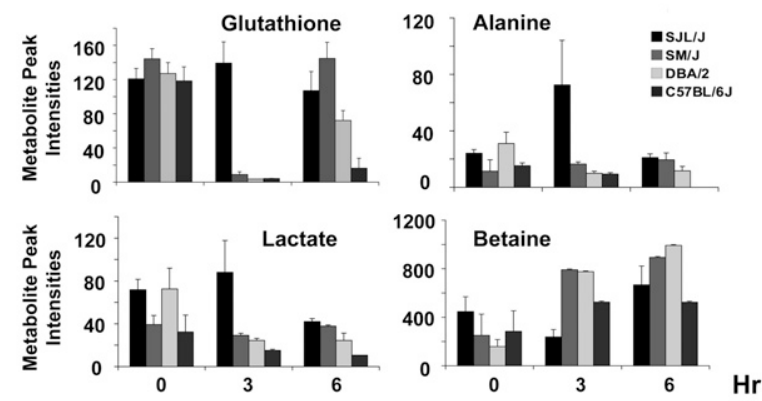

B
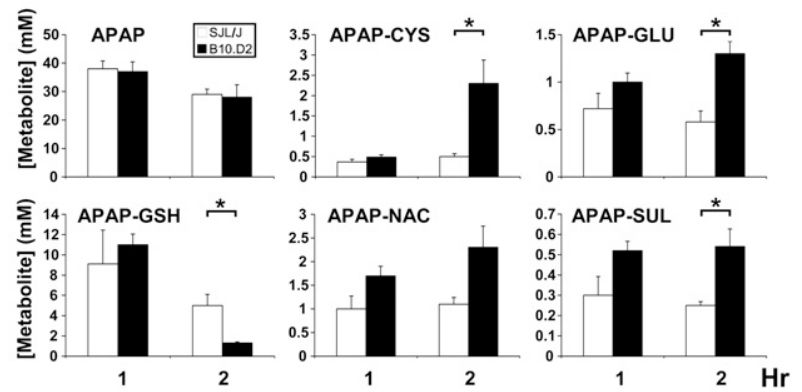

Figure 2. Metabolomic and pharmacokinetic analyses. (A) The abundance of 400 individual metabolite peaks in liver tissue obtained from four strains at 0,3 , and $6 \mathrm{~h}$ after APAP exposure was quantitatively analyzed by 2D NMR. At $3 \mathrm{~h}$, the abundance of GSH, alanine, lactate, and betaine exhibited a unique pattern in the resistant $S J \mathrm{~L} / \mathrm{J}$ mice, which differed from that in the three sensitive strains (DBA/2 I, C57BL/6], and SM/I). Each bar represents the average $\pm S D(N=3)$. (B) The amount of acetaminophen (APAP) and its metabolites in bile obtained from SJL/J or B10.D2 mice at 1 and $2 \mathrm{~h}$ after $300 \mathrm{mg} / \mathrm{kg}$ i.p. APAP was measured by LC/MS/MS analysis. Each bar represents the average \pm SE $(N=4-5)$ at the indicated time points, and asterisks $\left({ }^{*}\right)$ denote significant differences $(P<0.05)$ between two strains. Of note, there were no significant differences in APAP or any APAP-metabolite concentrations in bile at $1 \mathrm{~h}$; but there were significant differences in the concentration of several metabolites at $2 \mathrm{~h}$ after drug exposure. At that time, B10.D2 mice had fourfold lower APAP-GSH concentration than SJL/J mice, while there was a compensatory approximately fivefold increase in the concentration of the APAP-cysteine conjugate in B10.D2 mice relative to that in SJL/J mice. CYS, cysteine; GLU, glucoronate; NAC, N-acetylcysteine; and SUL, sulfate.

B10.D2 mice decreased $\sim 10$-fold and increased approximately fivefold, respectively, at the 2-h time point. Though both strains have similar initial patterns of APAP biotransformation, the susceptible strain had less ability to maintain hepatic GSH at a later time.

\section{Transcriptional and genetic analyses}

Gene expression changes occurring within these liver samples were simultaneously analyzed using microarrays. Within any one of these strains, there were a very large number of gene expression changes. By cross comparison, the resistant strain had 224 genes with an expression pattern that significantly differed (fold-change $>2$ and $P$-value $<0.01)$ from that in the three sensitive strains at $3 \mathrm{~h}$ after APAP exposure (Supplemental Table 3A). Even this was too large a number of genes to analyze, so we utilized the functional annotations within the Gene Ontology database (http://www. geneontology.org) to perform knowledge-based filtering of the 224 genes with an SJL/J-unique expression pattern. Only 20 of these genes were functionally annotated in the Gene Ontology database as being involved in metabolism. Moreover, only three genes (Pdk4, G6pc, and Bhmt2) were within pathways that affected the 4 endogenous metabolites identified by the metabolomic analyses (Table 1). Their expression pattern was confirmed by RT-PCR analyses (Supplemental Table 3B).

The pattern of genetic variation within the three differentially expressed genes identified by this integrative analysis was analyzed in 16 strains. None of the 49 single nucleotide polymorphisms (SNPs) within the $P d k 4$ gene altered its amino acid sequence, and 45 SNPs were only present in the SM/J strain. Since 14 sensitive strains and the resistant (SJL/J) strain had a similar pattern of genetic variation within the $P d k 4$ gene, we did not further pursue this gene candidate. In contrast, SNPs within G6pc (Ser291Leu, Ser292Leu) and Bhmt2 (Gly27Ser, Gly28Ser, Gln133Arg) induce multiple nonconserved amino acid substitutions. Furthermore, the pattern of genetic variation within the Bhmt2 (22 SNPs) and G6pc (11 SNPs) genes is organized into three distinct haplotypes among the 16 strains analyzed (Fig. 3). SJL/J mice share the Gly27Gly28Arg133 Bhmt2 haplotype with nine strains (129S1/ SvImJ, C3H/HeJ, NZB/BINJ, DBA/2J, LP/J, AKR/J, BALB/cJ, NZW/ $\mathrm{LacJ}$, and $\mathrm{SM} / \mathrm{J}$ ) that are sensitive to APAP-induced liver damage. SJL/J mice share the Ser291Ser292 G6pc haplotype with 11 other strains (A/J, A/HeJ, AKR/J, B10.D2, BALB/cJ, BALB/cByJ, C57BL/6J, MRL/MpJ, NZW/BINJ, SM/J, and LG/J). Bhmt2 and G6pc are located on different chromosomes, and neither has alleles that were uniquely present within resistant SJL/J mice. However, SJL/J mice do have a unique combination of Bhmt2 and G6pc haplotypes (Fig. $3)$. In addition, we noticed that the two most susceptible strains with the highest serum ALT levels (C57BL/6J and B10.D2) after APAP exposure shared a common Bhmt2 allele (Figs. 1A, 3).

\section{Bhmt2 is a candidate susceptible factor}

The APAP-induced changes in Bhmt2 mRNA and the decrease in hepatic betaine that were unique to $\mathrm{SJL} / \mathrm{J}$ mice were mechanistically intriguing. It has recently been demonstrated that BHMT2 is an SMM-specific homocysteine methyltransferase with a substrate specificity that is distinct from the two other homocysteine methylation enzymes (Bhmt, Mtr). BHMT2 has very low activity toward S-adenosylmethionine (SAM), and cannot use betaine as the methyl donor (Szegedi et al. 2008). Betaine is a required substrate for the BHMT-catalyzed remethylation of homocysteine to form methionine, which can then be converted to cysteine (Fig. 4).

Table 1. Pdk4, G6pc, and Bhmt2 mRNA are differentially expressed in SJL/J mice $3 \mathrm{~h}$ after acetaminophen administration

\begin{tabular}{|c|c|c|c|c|c|c|c|c|}
\hline & \multicolumn{2}{|c|}{ SJL/J } & \multicolumn{2}{|c|}{ SM/J } & \multicolumn{2}{|c|}{ DBA/2J } & \multicolumn{2}{|c|}{ C57BL/6J } \\
\hline & $\mathrm{Oh}$ & $3 \mathrm{~h}$ & $\mathrm{Oh}$ & $3 \mathrm{~h}$ & $\mathrm{Oh}$ & $3 \mathrm{~h}$ & $\mathrm{Oh}$ & $3 \mathrm{~h}$ \\
\hline $\begin{array}{l}\text { Pdk4 } \\
\text { G6pc } \\
\text { Bhmt2 }\end{array}$ & $\begin{array}{c}\mathbf{0 . 0 1 4} \pm \mathbf{0 . 0 0 3} \\
\mathbf{7 . 3 6} \pm \mathbf{1 . 3 4} \\
\mathbf{2 . 1 4} \pm \mathbf{0 . 1 4}\end{array}$ & $\begin{array}{c}\mathbf{0 . 0 0 3} \pm \mathbf{0 . 0 0 1} \\
\mathbf{1 . 1 0} \pm \mathbf{0 . 5 6} \\
\mathbf{0 . 7 5} \pm \mathbf{0 . 1 4}\end{array}$ & $\begin{array}{c}0.033 \pm 0.010 \\
3.58 \pm 0.48 \\
1.25 \pm 0.08\end{array}$ & $\begin{array}{c}0.019 \pm 0.004 \\
3.08 \pm 0.21 \\
1.02 \pm 0.07\end{array}$ & $\begin{array}{c}0.039 \pm 0.010 \\
4.68 \pm 0.54 \\
1.27 \pm 0.14\end{array}$ & $\begin{array}{c}0.027 \pm 0.015 \\
7.83 \pm 1.23 \\
1.34 \pm 0.31\end{array}$ & $\begin{array}{c}0.064 \pm 0.020 \\
7.34 \pm 1.61 \\
1.90 \pm 0.15\end{array}$ & $\begin{array}{c}0.039 \pm 0.010 \\
4.68 \pm 0.54 \\
1.27 \pm 0.14\end{array}$ \\
\hline
\end{tabular}

The expression levels were normalized relative to that of beta-actin. Each data point represents the average of relative expression levels \pm SE $(N=3)$. Boldface indicates differentially expressed PDK4, G6pc, and Bhmt2 in SJL/J mice $3 \mathrm{~h}$ after acetaminophen administration. 


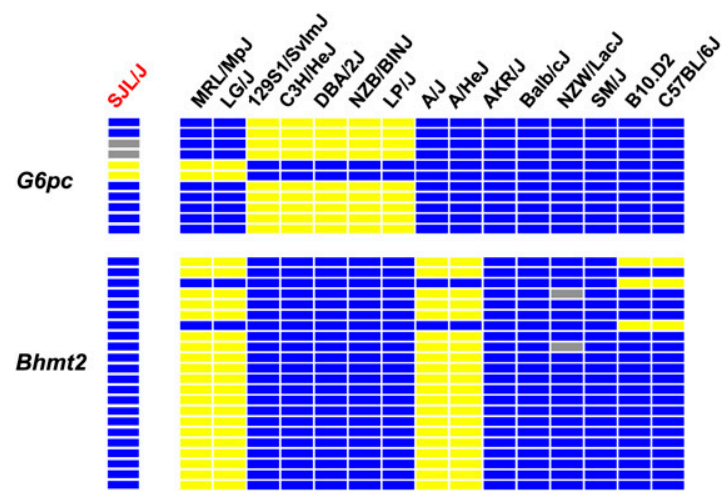

Figure 3. Haplotypes within Bhmt2 and G6pc for 16 inbred mouse strains. Each row represents one SNP and the color of each box represents the allele. A blue box denotes the common (or major) allele, while a yellow box is the less common (or minor) allele; a gray box indicates the allele is unknown. Groups of SNPs within Bhmt2 $(N=22)$ and G6pc $(N=11)$ form three distinct haplotypes for each gene across the 16 strains analyzed. Neither gene has a unique haplotype in the SJL/J strain, which is uniquely resistant to acetaminophen-induced liver injury.

Although BHMT can utilize SMM as a substrate in vitro with a very low affinity, it is unlikely that BHMT can use SMM in vivo where betaine will compete with SMM for its active site (Szegedi et al. 2008). If homocysteine is not remethylated, it can contribute to cysteine biosynthesis via the trans-sulfuration pathway. Since the cellular L-cysteine concentration is 10 -fold below that of the other two amino acids (L-glutamate, L-glycine) required for GSH biosynthesis, the amount of L-cysteine is a rate limiting factor (Griffith 1999). By this mechanism, a change in the abundance of a cofactor, a substrate, and/or an enzyme within the methionine/cysteine biosynthesis pathway could affect GSH production and resistance to drug-induced liver injury.

Therefore, liver extracts from eight inbred strains with three different Bhmt2 haplotypes were prepared to assess the enzyme activity of BHMT and BHMT2. All eight strains had comparable betainedependent BHMT activities, but only six strains with two different haplotypes had SMM-dependent BHMT2 activities (Table 2). Liver extracts prepared from the B10.D2 and C57BL/6J strains, which uniquely share the Ser27Gly28Arg133 haplotype, had minimal BHMT2 enzyme activity. Since Ser 27 is the only amino acid substitution unique to the B10.D2 and $\mathrm{C} 57 \mathrm{BL} / 6 \mathrm{~J}$ haplotype, this indicates that conversion of Gly 27 to Ser 27 caused a loss of hepatic BHMT2 enzyme activity. Analysis of the crystal structure of BHMT (Evans et al. 2002) demonstrated that the highly conserved $\mathrm{Asp}^{26}-\mathrm{Gly}^{27}$-Gly/Ala ${ }^{28}$ sequence is critical for homocysteine binding; small residues at positions 27 and 28 enable homocysteine to access the substrate-binding pocket (Collinsova et al. 2003). Thus, a substitution of Serine for Glycine at position 27 may alter substrate binding and cause a loss of enzyme activity. Since B10.D2 and C57BL/6J were among the strains that were most sensitive to APAP challenge (Fig. 1A), we hypothesized that Bhmt2 could be one of the susceptible factors affecting sensitivity to APAP-induced liver toxicity by influencing GSH biosynthesis. This possibility was strengthened by two other hepatic gene expression results. All four strains expressed comparable levels of mRNAs encoding two other homocysteine methyltransferases (Mtr, Bhmt) (data not shown), and none of the other genes involved in the GSH biosynthetic pathway had an SJL/J-specific gene expression pattern (Supplemental Table 4).

\section{Bhmt2 affects susceptibility to APAP toxicity}

The availability of two inbred strains with allelic variants that eliminate BHMT2 enzyme activity enabled the hypothesis that Bhmt2 is a susceptibility factor for APAP-induced liver toxicity to be tested in vivo. Within the methionine/glutathione biosynthesis pathway, methionine is converted to SAM, which is then used for cysteine and GSH biosynthesis. Administration of SAM was previously shown to attenuate APAP-induced hepatotoxicity and to preserve hepatic GSH levels in C57BL/6J mice (Bray et al. 1992; Valentovic et al. 2004; Terneus et al. 2007). Therefore, we analyzed

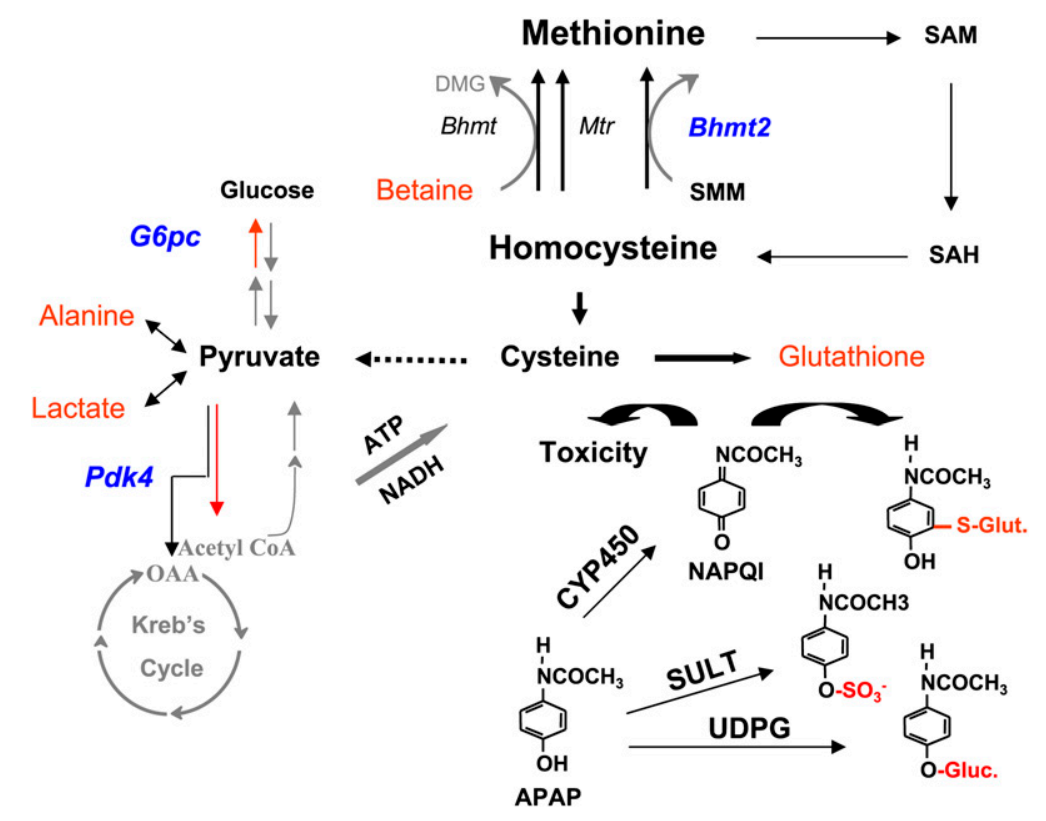

Figure 4. Metabolic pathways affecting sensitivity to acetaminophen (APAP)-induced hepatotoxicity. SJL/J mice that are uniquely resistant to APAP-induced liver toxicity had four endogenous metabolites (red text) with altered abundances, and three enzymes (blue text) had altered gene expression after drug exposure. The metabolic pathways encompassing these enzymes and endogenous metabolites are diagrammed. Cysteine biosynthesis is the rate-limiting step for glutathione (GSH) production. Alterations in Bhmt2 mRNA and betaine abundance that favor cysteine production would contribute to resistance to APAP-induced liver injury. Cysteine can be converted to pyruvate, which is in equilibrium with alanine and lactate. An increase in cysteine biosynthesis would explain why alanine and lactate were increased in the resistant mice. Reduced G6pc and Pdk4 will inhibit gluconeogenesis and favor pyruvate breakdown to increase cellular reducing capacity. These metabolic alterations might help SJL/J mice resist an APAP-induced toxic challenge. All three homocysteine methylation enzymes (Bhmt, Mtr, Bhmt2) are depicted in the figure. BHMT2 is an S-methyl-methionine (SMM)-specific methyltransferase and cannot use betaine as the methyl donor. Betaine is a required substrate for BHMT-catalyzed remethylation of homocysteine to form methionine. Sequential steps for SMM- and S-adenosyl methionine (SAM)-dependent methionine/GSH biosynthesis pathways are shown. APAP clearance by sulfation (SULT) or glucuronidation (UDPG), and cytochrome P450 (CYP450) mediated generation of NAPQI are depicted in the figure. Excess NAPQI would induce toxicity. 
Table 2. BHMT and BHMT2 activities of liver extracts from eight inbred strains with three different Bhmt2 haplotypes

\begin{tabular}{|c|c|c|c|c|c|}
\hline \multirow[b]{2}{*}{ Strain } & \multicolumn{3}{|c|}{ Haplotype } & \multirow[b]{2}{*}{ BHMT $^{a}$} & \multirow[b]{2}{*}{ BHMT $^{\mathrm{a}}$} \\
\hline & 27 & 28 & 133 & & \\
\hline MRL/MpJ & Gly & Ser & Glu & 66.8 & 6.58 \\
\hline $\mathrm{A} / \mathrm{J}$ & Gly & Ser & Glu & 65.2 & 8.08 \\
\hline $\mathrm{A} / \mathrm{HeJ}$ & Gly & Ser & Glu & 72.2 & 8.47 \\
\hline $\mathrm{SM} / \mathrm{J}$ & Gly & Gly & Arg & 128 & 9.31 \\
\hline $129 \mathrm{~S} 1 / \mathrm{SvImJ}$ & Gly & Gly & Arg & 69.0 & 8.03 \\
\hline DBA/2J & Gly & Gly & Arg & 92.3 & 7.39 \\
\hline C57BL/6] & Ser & Gly & Arg & 88.4 & 0.34 \\
\hline B10.D2 & Ser & Gly & Arg & 90.7 & 0.10 \\
\hline
\end{tabular}

Each data point is the average enzyme activity measured in two independent experiments, and is expressed in units per milligram protein. Boldface indicates Ser 27 abolished BHMT2 activity.

${ }^{a}$ One unit equals 1 pmol of methyl donor substrates consumed per hour.

the effect that coadministration of two metabolites (SAM and SMM), which act on sequential steps in the methionine/GSH biosynthesis pathway, had on APAP-induced liver toxicity in strains with intact or deficient BHMT2 activity (Figs. 4, 5A). If BHMT2 affects susceptibility, SMM coadministration should protect against APAP-induced liver damage in strains with intact BHMT2 enzyme activity, but should not have an effect in strains with inactive BHMT2 enzyme activity. Since SAM acts just downstream of the BHMT2-catalyzed step, it should have a protective effect in all strains.

SMM coadministration had a significant protective effect in DBA/2J $(P$-value $<0.01)$, but not in B10.D2 $(P$-value $=0.973)$ or C57BL/6J mice $(P$-value $=0.999)($ Fig. $5 B)$. The median serum ALT in SMM-treated mice was threefold below that of control APAPtreated DBA/2J mice. In contrast, SAM had a very significant protective effect in mice with active $(\mathrm{DBA} / 2 \mathrm{~J}, P$-value $<0.001)$ or inactive (B10.D2, $P$-value $<0.001 ;$ C57BL/6J, $P$-value $<0.0001$ ) BHMT2 enzymes. Thus, SMM coadministration had the predicted strain-specific effect in reducing the extent of APAP-induced liver toxicity. Of note, SMM coadministration caused a paradoxical increase in liver toxicity in the Bhmt2 deficient strains. This effect may be due to BHMT having a fivefold lower catalytic efficiency when SMM is the substrate (Szegedi et al. 2008), which is of increased importance in mice that lack BHMT2 activity. In these individuals, an increased amount of ingested SMM would compete with betaine as a substrate for BHMT in this setting.

To precisely localize the site of SMM's protective effect, the hepatic concentration of GSH and methionine was also measured in two strains. APAP induced a $>90 \%$ decrease in liver GSH concentration at $3 \mathrm{~h}$ in $\mathrm{A} / \mathrm{J}$ and $\mathrm{C} 57 \mathrm{BL} / 6 \mathrm{~J}$ mice. Consistent with its proposed site of action, SMM coadministration caused a significant increase in hepatic GSH $(P<0.001, \sim 100 \%)$ and methionine $(P<$ 0.001 , approximately threefold) in $\mathrm{A} / \mathrm{J}$ mice, which have active BHMT2 enzyme; but not in C57BL/6J mice that have inactive enzymes (Fig. 5C). These strain-specific effects on hepatic metabolite levels confirmed that SMM affected the BHMT2-catalyzed reaction in methionine/GSH biosynthesis pathways in vivo.

\section{Discussion}

This integrative genetic, transcriptional, and metabolomic analysis plus knowledge-based filtering led to the finding that Bhmt 2 is one of several genetic factors affecting susceptibility to APAP-induced liver toxicity. Coadministration of a BHMT2-specific methyl donor
(SMM) only protected those strains with intact BHMT2 enzyme activity from APAP-induced toxicity; the decrease in serum ALT and the increase in hepatic methionine and GSH levels confirmed its site-specific protective effect. Pharmacokinetic profiling also confirmed that the resistant strain had a superior ability to maintain hepatic GSH levels after APAP exposure. Interestingly, gene expression criteria were used to identify Bhmt2 as a candidate genetic factor; but the subsequent analysis demonstrated that a nonsynonymous Gly27Ser polymorphism, which determined whether an active or inactive enzyme was produced, was the causative genetic change. All six strains with the Gly 27 allele that were analyzed had comparable and significant levels of hepatic BHMT2 enzyme activity, while both strains with a Ser 27 allele had inactive enzyme. It is therefore likely that reduced Bhmt 2 mRNA expression in SJL/J mice after APAP exposure results from another mechanism, which is likely to be transcriptional inhibition induced by metabolites within the methionine/glutathione biosynthesis pathway. Administration of exogenous SAM has been shown to decrease BHMT mRNA transcription (Castro et al. 2001;

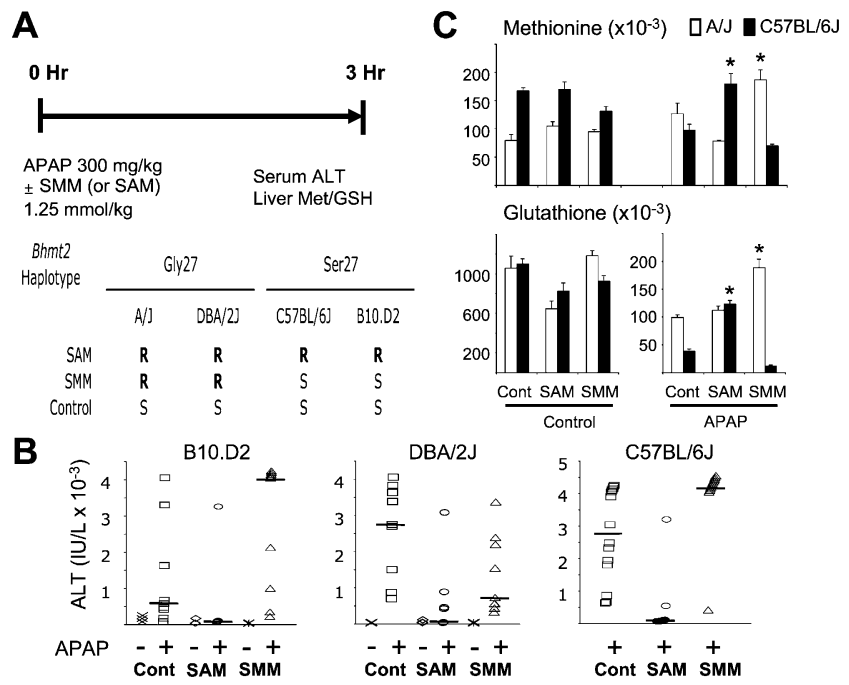

Figure 5. (A) Diagram of an experiment assessing the strain-specific effect of SMM or SAM coadministration on APAP-induced liver toxicity. Hepatic glutathione (GSH) and methionine levels and the serum ALT were measured $3 \mathrm{~h}$ after APAP administration to inbred strains. If BHMT2 activity affected sensitivity to APAP-induced liver injury, coadministration of a BHMT2-specific substrate (SMM) should have a protective effect in strains with active BHMT2 enzyme (Gly 27 allele), but not in strains with inactive (Ser 27 allele) enzyme. In contrast, SAM, which affects GSH biosynthesis at a step distal to BHMT2, should have a protective effect in all strains. Based upon the Bhmt2 allele, the R and $S$ in the lower panel indicate whether SMM coadministration should have a protective (rescue, R) effect, or not have a beneficial (susceptible, S) effect on APAP-induced liver toxicity, respectively. (B) The serum ALT level was measured $3 \mathrm{~h}$ after treatment with vehicle, SAM, or SMM plus either vehicle or APAP. Nine or more mice were in each APAP-treated group. The horizontal line indicates the median value for each group, and $P$-values assessing whether SAM or SMM coadministration decreased the serum ALT levels relative to control were calculated. For DBA/2J mice, the $P$-values for the effect of SAM and SMM coadministration were 0.00058 and 0.0087 , respectively. The effect of SAM and SMM coadministration in B10.D2 mice had $P$-values of 0.00029 and 0.9735 , respectively. C57BL/6J mice had similar $P$-values (0.0000002 for SAM; 0.9985 for SMM). (C) C57BL/6] or A/J mice were treated with vehicle, SAM, or SMM plus either vehicle or APAP. Liver samples were obtained $3 \mathrm{~h}$ after dosing and the amount of GSH and methionine were determined by $\mathrm{LC} / \mathrm{MS} / \mathrm{MS}$ analysis. The data represent the average \pm SE for $N=$ three samples. An asterisk $\left({ }^{*}\right)$ indicates a $P$-value $<$ 0.001 relative to the control-APAP treated mice by Student's $t$-test.

\section{Genome Research www.genome.org}


Ou et al. 2007). The increased glutathione and decreased betaine levels indicate that the flux through the methionine biosynthesis pathway, which produces SAM, was increased in SJL/J mice after APAP exposure. Therefore, Bhmt2 is a novel, diet-dependent, genetic factor that affects methionine and glutathione biosynthesis, and susceptibility to APAP-induced liver injury.

The extent of APAP-induced liver injury was similarly analyzed in 36 inbred mouse strains by other investigators, and this analysis led to the finding that genetic variation in human CD44 contributed to susceptibility to APAP-induced liver toxicity (Harrill et al. 2009). It is not unexpected that polymorphisms within geneeffecting immunological responses could affect susceptibility. There were differences in the serum ALT levels among the inbred strains analyzed here and in the Harrill et al. (2009) study. However, this could result from the different dosing regimens used in the two studies; Harrill and colleagues used intragastric feeding, while we used intraperitoneal injection. Despite these differences, both studies demonstrate that analysis of differences in susceptibility to APAP-induced liver injury among inbred mouse strains can provide important information.

SMM is a very ubiquitous and abundant constituent of the free amino acid pool in most flowering plants (Mudd and Datko 1990; Paquet et al. 1995; Augspurger et al. 2005) that is universally ingested in the diet. Methionine metabolism in plants differs from that in mammalian organisms by involving SMM, which is the major metabolite used to transport sulfated amino acids (Bourgis et al. 1999). SMM was $80 \%$ of the total amount of ${ }^{35}$ S-labeled material in phloem of a wheat plant (Bourgis et al. 1999). The absence of the required methyltransferase renders mammals unable to synthesize SMM from L-methionine, yet they clearly obtain SMM in their diet (Mudd and Datko 1990). The protective effect of SMM suggests a novel strategy that could be used to prevent APAPinduced liver injury. It is likely that the methionine/GSH biosynthetic pathway in humans resembles that in mouse strains with an active BHMT2 enzyme. Recently, multiple polymorphisms within BHMT $(n=25)$ and BHMT2 $(n=39)$ have been identified in human populations, including four SNPs that alter the predicted amino acid sequence of each of these two proteins (Li et al. 2008). Given the extensive genetic variation in humans, it is possible that genetic variation within this pathway could also affect susceptibility to drug-induced liver injury in humans. The genetic complexity of this drug response is indicated by the fact that all susceptible mouse strains, except C57BL/6J and B10.D2, have functional Bhmt 2 alleles. Thus, Bhmt 2 is only one of several genetic factors affecting susceptibility. It is worthwhile to continue the search for other genetic susceptibility factors for APAP-induced liver injury.

$\mathrm{N}$-acetylcysteine is the most effective agent for treatment of ongoing APAP-liver toxicity (Chun et al. 2009). However, N-acetylcysteine has a very unpleasant taste and odor, is poorly tolerated after oral administration, and can induce nausea and vomiting. In contrast, SMM does not have these properties, which makes it conceivable that it could be prospectively ingested to prevent APAP-induced liver injury. SMM was originally referred to as "vitamin U" and has been widely used in Chinese herbal medicine for treatment of gastrointestinal diseases and in clinical studies dating back to the 1950s (Cheney 1954). It is with some irony that these genetic, genomic, and metabolomic analyses in mice have uncovered the mechanism of action of a metabolite that is already widely used in Chinese herbal medicine. Since SMM is a widely available dietary supplement, it is of importance to further test its ability to prevent APAP-induced liver injury. Nevertheless, this integrative analysis of metabolomic, transcriptomic, and genetic data, along with knowledge-based filtering, successfully identified Bhmt2 as a host factor protecting against APAP-induced liver injury. Neither metabolomic nor transcriptomic profiling alone would have been sufficient to pinpoint causative genes. The list of APAP-induced changes in hepatic metabolites or gene expression within any one strain was quite large and un-manageable, which explains why so many different factors have been implicated in previous studies of APAP toxicity. It is also noteworthy that the inbred mouse strain $(\mathrm{C} 57 \mathrm{BL} / 6 \mathrm{~J})$ that is most commonly used in studies of APAP toxicity is unusual in that it lacks BHMT2 enzyme activity. Only through applying this type of integrative analysis could we gain insight into the role that Bhmt2 might play, which led to a testable hypothesis that could address a major public health problem.

\section{Methods}

\section{Animal husbandry and drug treatment}

All animal experiments followed protocols that were approved by Roche Palo Alto Institutional Animal Care and Use Committee. The mice were housed in a pathogen-free environment with a $12 \mathrm{~h}: 12 \mathrm{~h}$ light:dark cycle and had access to food and water ad libitum. Before APAP exposure, mice were fasted overnight $(>16 \mathrm{~h}$ ) to uniformly deplete hepatic glutathione stores (Bartolone et al. 1987). After APAP or vehicle challenges, mice regained free access to food and water. According to the protocol approved for the morbidity experiments, veterinarians that were blinded to whether the mice were administered vehicle or APAP periodically evaluated the mice during these experiments. Based on guidelines for humane endpoints, mice that exhibited significant weight loss, lethargy, dehydration, hunched body posture, or were cold to touch were identified as moribund by the veterinarians and were euthanized. 7-8-wk old male mice were obtained from Jackson Laboratory and acclimatized for an additional week before use. The following 16 inbred mouse strains were studied: SJL/J, LG/J, BALB/ cJ, DBA/2J, A/J, AKR/J, A/HeJ, 129S1/SvImJ, B10.D2-H2/oSNJ (abbreviated as B10.D2 elsewhere), C57BL/6J, NZW/LacJ, NZB/BINJ, MRL/MpJ, C3H/HeJ, LP/J, and SM/J. PBS (pH 7.4) was used as drug vehicles. Acetaminophen (Voigt Global Distribution Inc.) was dissolved in heat, while S-adenosyl methionine (Sigma) or S-methyl methionine was prepared at room temperature. Solutions were sterilized through $0.2-\mu \mathrm{m}$ filters before use. Drugs were administered by i.p. bolus injection.

\section{Tissue collection and analysis}

Mice were sacrificed by $\mathrm{CO}_{2}$ inhalation or cervical dislocation. Blood was collected by cardiac puncture; urine was directly drained from voluntary action; bile was withdrawn from gall bladders with insulin needles. Liver samples were either fixed in buffered formalin for histology or snap frozen in liquid nitrogen before homogenization for LC/MS/MS or NMR analyses. Alanine aminotransferase (ALT) activity was measured at the Marshfield Clinic Laboratories. Hematoxylin and eosin staining upon $10-\mu \mathrm{m}$-thick hepatic sections was performed by standard methods. APAP metabolites, hepatic methionine/glutathione analysis by LC/MS/MS, and metabolomic profiling by 2D NMR were performed as described in the Supplemental material.

\section{SNP and gene expression analysis}

The methods for murine SNP discovery and for haplotype block construction have been published elsewhere (Liao et al. 2004; Wang et al. 2005). The online database is located at http:// 
mousesnp.roche.com/. Gene expression analysis was performed as described in the Supplemental material.

\section{Hepatic BHMT and BHMT2 activity}

The transfer of radio-labeled methyl groups to homocysteine was assayed as described previously (Garrow 1996; Jakubowski 2004). Substrates for BHMT and BHMT2 were radio-labeled betaine $(250 \mu \mathrm{M}, 0.25 \mu$ Curie $)$ and SMM $(250 \mu \mathrm{M}, 0.28 \mu$ Curie), respectively. BHMT2 assays were performed in the presence of $62.5 \mu \mathrm{M}$ carboxybutylhomocysteine, which is a potent inhibitor of BHMT (Castro et al. 2004). Radio-labeled SMM was prepared as described in the Supplemental material.

\section{Statistical methods}

Wilcoxon rank sum test was used in Figure 5B because serum alanine aminotransferase was not normally distributed. Student's $t$-test was used elsewhere.

\section{Acknowledgments}

Y-Y.G., P.L. and E.F. were supported by a grant (1 R01 GM06888501A1) from the NIGMS awarded to G.P. This work was also supported by a grant (1 R01 DK52501) from the NIDDK awarded to T.A.G. We thank Christopher Smith and Pamela Mclawhon for expert animal care and technical assistance; Alberto Lapresca and Earl Meierhenry for help with analysis of liver tissues; and David Clark, Bob Lewis, Richard Hanson, Lance Pohl, Adrien Fretland, and Kaushik Chakravarty for helpful discussions.

\section{References}

Augspurger NR, Scherer CS, Garrow TA, Baker DH. 2005. Dietary S-methylmethionine, a component of foods, has choline-sparing activity in chickens. J Nutr 135: 1712-1717.

Bai J, Cederbaum AI. 2004. Adenovirus mediated overexpression of CYP2E1 increases sensitivity of HepG2 cells to acetaminophen induced cytotoxicity. Mol Cell Biochem 262: 165-176.

Bartolone JB, Sparks K, Cohen SD, Khairallah EA. 1987. Immunochemical detection of acetaminophen-bound liver proteins. Biochem Pharmacol 36: $1193-1196$.

Bessems JG, Vermeulen NP. 2001. Paracetamol (acetaminophen)-induced toxicity: Molecular and biochemical mechanisms, analogues and protective approaches. Crit Rev Toxicol 31: 55-138.

Bhave VS, Donthamsetty S, Latendresse JR, Mehendale HM. 2008. Inhibition of cyclooxygenase- 2 aggravates secretory phospholipase A2-mediated progression of acute liver injury. Toxicol Appl Pharmacol 228: $239-246$.

Bourgis F, Roje S, Nuccio ML, Fisher DB, Tarczynski MC, Li C, Herschbach C, Rennenberg H, Pimenta MJ, Shen TL, et al. 1999. S-methylmethionine plays a major role in phloem sulfur transport and is synthesized by a novel type of methyltransferase. Plant Cell 11: 1485-1498.

Bray GP, Tredger JM, Williams R. 1992. S-adenosylmethionine protects against acetaminophen hepatotoxicity in two mouse models. Hepatology 15: $297-301$.

Castro CC, Breksa AP, Salisbury EM, Garrow TA, Milstein S, Kapatos G, Levine R, Shane B. 2001. Betaine-homocysteine S-methyltransferase transcription is inhibited by S-adenosylmethionine. In Chemistry and Biology of Pteridines and Folates: Proceedings of the 12th International Symposium on Pteridines and Folates, pp. 549-562. Kluwer, Norwell, MA

Castro C, Gratson AA, Evans JC, Jiracek J, Collinsova M, Ludwig ML, Garrow TA. 2004. Dissecting the catalytic mechanism of betaine-homocysteine S-methyltransferase by use of intrinsic tryptophan fluorescence and sitedirected mutagenesis. Biochemistry 43: 5341-5351.

Chen C, Hennig GE, Manautou JE. 2003. Hepatobiliary excretion of acetaminophen glutathione conjugate and its derivatives in transportdeficient (TR-) hyperbilirubinemic rats. Drug Metab Dispos 31: 798-804

Cheney G. 1954. Vitamin U concentrate therapy of peptic ulcer. Am I Gastroenterol 21: 230-250.

Chun LJ, Tong MJ, Busuttil RW, Hiatt JR. 2009. Acetaminophen hepatotoxicity and acute liver failure. J Clin Gastroenterol 43: 342-349.
Collinsova M, Castro C, Garrow TA, Yiotakis A, Dive V, Jiracek J. 2003. Combining combinatorial chemistry and affinity chromatography: Highly selective inhibitors of human betaine: Homocysteine S-methyltransferase. Chem Biol 10: 113-122.

Dai G, He L, Chou N, Wan YJ. 2006. Acetaminophen metabolism does not contribute to gender difference in its hepatotoxicity in mouse. Toxicol Sci 92: $33-41$.

Duda RO, Hart PE, Stork DG. 2001. Pattern classification, 2nd ed. WileyInterscience, New York.

Evans JC, Huddler DP, Jiracek J, Castro C, Millian NS, Garrow TA, Ludwig ML. 2002. Betaine-homocysteine methyltransferase: Zinc in a distorted barrel. Structure 10: 1159-1171.

Garrow TA. 1996. Purification, kinetic properties, and cDNA cloning of mammalian betaine-homocysteine methyltransferase. J Biol Chem 271: 22831-22838.

Griffith OW. 1999. Biologic and pharmacologic regulation of mammalian glutathione synthesis. Free Radic Biol Med 27: 922-935.

Guo GL, Moffit JS, Nicol CJ, Ward JM, Aleksunes LA, Slitt AL, Kliewer SA, Manautou JE, Gonzalez FJ. 2004. Enhanced acetaminophen toxicity by activation of the pregnane X receptor. Toxicol Sci 82: 374-380.

Harrill AH, Watkins PB, Su S, Ross PK, Harbourt DE, Stylianou IM, Boorman GA, Russo MW, Sackler RS, Harris SC, et al. 2009. Mouse population-guided resequencing reveals that variants in CD44 contribute to acetaminopheninduced liver injury in humans. Genome Res 19: 1507-1515.

Hogaboam CM, Bone-Larson CL, Steinhauser ML, Matsukawa A, Gosling J, Boring L, Charo IF, Simpson KJ, Lukacs NW, Kunkel SL. 2000. Exaggerated hepatic injury due to acetaminophen challenge in mice lacking C-C chemokine receptor 2. Am J Pathol 156: 1245-1252.

Ishida Y, Kondo T, Ohshima T, Fujiwara H, Iwakura Y, Mukaida N. 2002. A pivotal involvement of IFN- $\gamma$ in the pathogenesis of acetaminopheninduced acute liver injury. FASEB J 16: 1227-1236.

Ishida Y, Kondo T, Tsuneyama K, Lu P, Takayasu T, Mukaida N. 2004. The pathogenic roles of tumor necrosis factor receptor p55 in acetaminophen-induced liver injury in mice. J Leukoc Biol 75: 59-67.

Jakubowski H. 2004. Molecular basis of homocysteine toxicity in humans. Cell Mol Life Sci 61: 470-487.

James LP, Mayeux PR, Hinson JA. 2003. Acetaminophen-induced hepatotoxicity. Drug Metab Dispos 31: 1499-1506.

Jia Y, Guo GL, Surapureddi S, Sarkar J, Qi C, Guo D, Xia J, Kashireddi P, Yu S, Cho YW, et al. 2005. Transcription coactivator peroxisome proliferatoractivated receptor-binding protein/mediator 1 deficiency abrogates acetaminophen hepatotoxicity. Proc Natl Acad Sci 102: 12531-12536.

Li F, Feng Q, Lee C, Wang S, Pelleymounter LL, Moon I, Eckloff BW, Wieben ED, Schaid DJ, Yee V, et al. 2008. Human betaine-homocysteine methyltransferase (BHMT) and BHMT2: Common gene sequence variation and functional characterization. Mol Genet Metab 94: 326-335.

Liao G, Wang J, Guo J, Allard J, Cheng J, Ng A, Shafer S, Puech A, McPherson JD, Foernzler D, et al. 2004. In silico genetics: Identification of a functional element regulating H2-E $\alpha$ gene expression. Science 306: 690-695.

Limaye PB, Apte UM, Shankar K, Bucci TJ, Warbritton A, Mehendale HM. 2003. Calpain released from dying hepatocytes mediates progression of acute liver injury induced by model hepatotoxicants. Toxicol Appl Pharmacol 191: 211-226.

Limaye PB, Bhave VS, Palkar PS, Apte UM, Sawant SP, Yu S, Latendresse JR, Reddy JK, Mehendale HM. 2006. Upregulation of calpastatin in regenerating and developing rat liver: Role in resistance against hepatotoxicity. Hepatology 44: 379-388.

Liu ZX, Govindarajan S, Kaplowitz N. 2004. Innate immune system plays a critical role in determining the progression and severity of acetaminophen hepatotoxicity. Gastroenterology 127: 1760-1774.

Masson MJ, Carpenter LD, Graf ML, Pohl LR. 2008. Pathogenic role of natural killer T and natural killer cells in acetaminophen-induced liver injury in mice is dependent on the presence of dimethyl sulfoxide. Hepatology 48: 889-897.

Masubuchi Y, Sugiyama S, Horie T. 2009. Th1/Th2 cytokine balance as a determinant of acetaminophen-induced liver injury. Chem Biol Interact 179: $273-279$.

Meister A, Anderson ME. 1983. Glutathione. Annu Rev Biochem 52: 711-760.

Mudd SH, Datko AH. 1990. The S-methylmethionine cycle in Lemna paucicostata. Plant Physiol 93: 623-630.

Ou X, Yang H, Ramani K, Ara AI, Chen H, Mato JM, Lu SC. 2007. Inhibition of human betaine-homocysteine methyltransferase expression by S-adenosylmethionine and methylthioadenosine. Biochem J 401: 87-96.

Paquet L, Lafontaine PJ, Saini HS, James F, Hanson AD. 1995. Evidence en faveur de la presence du 3-dimethylsulfoniopropionate chez unelarge gamme d'Angiospermes. Can J Bot 73: 1889-1896.

Perkins JD. 2006. Acetaminophen sets records in the United States: Number 1 analgesic and number 1 cause of acute liver failure. Liver Transpl 12: 682-683.

Qiu Y, Benet LZ, Burlingame AL. 1998. Identification of the hepatic protein targets of reactive metabolites of acetaminophen in vivo in mice using

\section{Genome Research}


two-dimensional gel electrophoresis and mass spectrometry. J Biol Chem 273: $17940-17953$.

Reilly TP, Bourdi M, Brady JN, Pise-Masison CA, Radonovich MF, George JW, Pohl LR. 2001. Expression profiling of acetaminophen liver toxicity in mice using microarray technology. Biochem Biophys Res Commun 282: 321-328.

Ruepp SU, Tonge RP, Shaw J, Wallis N, Pognan F. 2002. Genomics and proteomics analysis of acetaminophen toxicity in mouse liver. Toxicol Sci 65: $135-150$.

Szegedi SS, Castro CC, Koutmos M, Garrow TA. 2008. Betaine-homocysteine S-methyltransferase-2 is an S-methylmethionine-homocysteine methyltransferase. J Biol Chem 283: 8939-8945.

Terneus MV, Kiningham KK, Carpenter AB, Sullivan SB, Valentovic MA. 2007. Comparison of S-adenosyl-L-methionine and N-acetylcysteine protective effects on acetaminophen hepatic toxicity. J Pharmacol Exp Ther 320: 99-107.
Valentovic M, Terneus M, Harmon RC, Carpenter AB. 2004. S-adenosylmethionine (SAMe) attenuates acetaminophen hepatotoxicity in C57BL/6 mice. Toxicol Lett 154: 165-174.

Wang J, Liao G, Usuka J, Peltz G. 2005. Computational genetics: From mouse to human? Trends Genet 21: 526-532.

Welch KD, Wen B, Goodlett DR, Yi EC, Lee H, Reilly TP, Nelson SD, Poh LR. 2005. Proteomic identification of potential susceptibility factors in drug-induced liver disease. Chem Res Toxicol 18: 924 933.

Zheng M, Lu P, Liu Y, Pease J, Usuka J, Liao G, Peltz G. 2007. 2D NMR metabonomic analysis: A novel method for automated peak alignment. Bioinformatics 23: 2926-2933.

Received June 12, 2009; accepted in revised form September 24, 2009. 


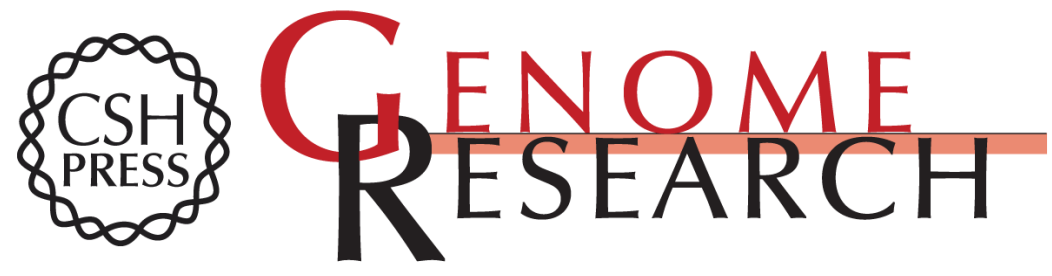

\section{An integrative genomic analysis identifies Bhmt2 as a diet-dependent genetic factor protecting against acetaminophen-induced liver toxicity}

Hong-Hsing Liu, Peng Lu, Yingying Guo, et al.

Genome Res. 2010 20: 28-35 originally published online November 18, 2009

Access the most recent version at doi:10.1101/gr.097212.109

\footnotetext{
Supplemental http://genome.cshlp.org/content/suppl/2009/10/14/gr.097212.109.DC1

Material

Related Content Mouse population-guided resequencing reveals that variants in CD44

contribute to acetaminophen-induced liver injury in humans

Alison H. Harrill, Paul B. Watkins, Stephen Su, et al.

Genome Res. September , 2009 19: 1507-1515

References This article cites 45 articles, 13 of which can be accessed free at:

http://genome.cshlp.org/content/20/1/28.full.html\#ref-list-1

Articles cited in:

http://genome.cshlp.org/content/20/1/28.full.html\#related-urls

\section{License}

Email Alerting Receive free email alerts when new articles cite this article - sign up in the box at the Service top right corner of the article or click here.
}

\section{Affordable, Accurate Sequencing.}

